\title{
Construcción del cuestionario de estrategias de afrontamiento al ciberacoso en adolescentes del departamento de Puno, 2021
}

\author{
Construction of the questionnaire on coping strategies for cyberbullying in \\ adolescents from the department of Puno, 2021
}

\author{
Janett Rosaly Cayo Caceres ${ }^{1}$, Heber Mario Pari Betancur², Eddy Wildmar Aquize Anco ${ }^{3}$
}

\begin{abstract}
RESUMEN
Objetivo: analizar las propiedades psicométricas del cuestionario de estrategias de afrontamiento al ciberacoso. Metodología: el estudio se realizó en una muestra de 250 adolescentes entre 14 y 17 años de edad para la prueba piloto y 300 adolescentes entre 12 a 17 años de edad entre varones y mujeres, la recolección de datos se realizó de manera virtual por medio de la plataforma Google formulario. Resultado: el cuestionario de estrategias de afrontamiento al ciberacoso en su versión final, posee un índice de validez ( $V=.98)$ que se obtuvo gracias a la evaluación de seis profesionales expertos y teniendo 4 criterios de evaluación (Claridad, Contexto, Congruencia y Dominio del constructo). En cuanto a la validez de contenido, se logró a través de las pruebas de KMO y esfericidad de Bartlett cuyos valores fueron $(\mathrm{KMO}=.876 ; \mathrm{P}<.05)$ lo que se traduciría en que instrumento es apto para el análisis factorial confirmatorio (AFC), lo cual sugiere que el instrumento debe poseer un contenido distribuido en 2 factores que explican el $41,7 \%$ de la varianza acumulada, se realizó un análisis más detallado mediante las ecuaciones estructuras, en la cual se halló RMSEA =0,077 mayor al ,005; $\mathrm{CFI}=0,882, \mathrm{Y}$ UN TLI $=0,860$ ambos resultados demuestran un adecuado ajuste. Respecto a la confiabilidad de instrumento a través del alfa de Cronbach este alcanzó un (.873), así mismo se analizó la fiabilidad de las dos dimensiones del cuestionario mediante el cálculo del coeficiente Omega de McDonald's (evaluación las amenazas y evaluación de afrontamiento) obteniendo una fiabilidad de .957 y. 851 respectivamente lo que indicaría que instrumento posee una confiabilidad global adecuada. Conclusión: el instrumento evidencia una buena consistencia interna y validez de constructo.
\end{abstract}

Palabras clave: Ciberacoso; estrategias de afrontamiento; redes sociales; adolescentes

\section{ABSTRACT}

Objective: to analyze the psychometric properties of the cyberbullying coping strategies questionnaire. Methodology: the study was carried out in a sample of 250 adolescents between 14 and 17 years of age for the pilot test and 300 adolescents between 12 and 17 years of age between men and women, the data collection was carried out virtually by means of the Google form platform. Result: the questionnaire on cyberbullying coping strategies in its final version has a validity index $(\mathrm{V}=.98)$ that was obtained thanks to the evaluation of six expert professionals and having 4 evaluation criteria (Clarity, Context, Congruence and Construct domain). Regarding content validity, it was achieved through the $\mathrm{KMO}$ and Bartlett's sphericity tests whose values were $(\mathrm{KMO}=.876 ; \mathrm{P}<.05)$ which would translate into which instrument is suitable for confirmatory factor analysis (AFC), which suggests that the instrument must have a content distributed in 2 factors that explain $41.7 \%$ of the accumulated variance, a more detailed analysis was carried out using the structural equations, in which RMSEA $=0.077$ greater than the, $005 ; \mathrm{CFI}=0.882$, and $\mathrm{UN} \mathrm{TLI}=0.860$, both results show an adequate fit. Regarding the reliability of the instrument through Cronbach's alpha, this reached (.873), likewise the reliability of the two dimensions of the questionnaire was analyzed by calculating the McDonald's

\footnotetext{
'Universidad Peruana Unión, Juliaca, Perú. Orcid ID: 0000-0001-7559-1448

${ }^{2}$ Universidad Peruana Unión, Juliaca, Perú.

Orcid ID: 0000-0002-1502-7279

${ }^{3}$ Universidad Peruana Unión, Juliaca, Perú.

Orcid ID: 0000-0002-4820-9570
} 
Omega coefficient (evaluation of threats and evaluation of coping) obtaining a reliability of .957 and. 851 respectively, which would indicate which instrument has adequate global reliability. Conclusion: the instrument shows good internal consistency and construct validity

Keywords: Cyberbullying; coping strategies; social networks; teenagers

\section{INTRODUCCIÓN}

Los celulares e internet, se han transformado de un tiempo a otro en herramientas peligrosas, para agraviar, coaccionar, intimidar a otras personas (Álvarez-García et al., 2011).

A medida que van pasando los años, el internet está cada vez más al alcance de los niños y adolescentes el cual, se hace parte de su vida diaria. El portal web Sexta (2019) reporta un evidente crecimiento en los casos de Ciberacoso. Según la encuesta realizada en 30 países por la UNICEF, 1 de cada 3 adolescentes, menciona haber sido víctima de ciberacoso. Según la directora ejecutiva de la UNICEF, menciona: "En todo el mundo los jóvenes nos están diciendo que están siendo intimidados por internet, que eso está afectando su educación y que quieren que se termine" (Henrietta-Fore, 2019).

Según la Organización Mundial de Salud (OMS, 2016) uno de cada cuatro casos de acoso, se da de manera cibernética, y esto va en aumento a partir de los 13 años de edad ya que, uno de cada tres víctimas de ciberacoso tiene dicha edad; asimismo, revela que el género más afectado es el femenino. Según el portal Web Hiperderecho, entre febrero del 2018 y junio del 2020, se han registrado 2580 denuncias de ciberacoso y violencia online. De ellos, 2331 casos fueron denunciados por las víctimas y 349 fueron denunciados por informantes (Albornoz, 2020).

La división de Investigación de Alta Tecnología (DIVINDAT) de la Policía Nacional del Perú, indicó que el $77 \%$ de escolares entre 9 a 14 años, admitió ser víctima de acoso sexual por redes sociales y juegos en línea (Perú21, 2016). Por otra parte, el Ministerio de Educación (MINEDU) realizó un estudio entre el 2014 al 2016 por el portal SíseVe, en el que se evidencia que el $62 \%$ de los estudiantes encuestados aseguraron haber sido testigos de hostigamiento en contra de sus compañeros, y el $47.2 \%$ señaló que la agresión se dio a través del internet (Perú21, 2017).
Estos casos provocan impactos negativos en las victimas, y permite que el acosador se mantenga en el anonimato. Las víctimas del ciberacoso pueden sufrir de depresión, baja autoestima, mala conducta, bajo rendimiento escolar, etc. (Mayo, Benjamin, \& Ang, 2012).

Chan y Wong (2017) realizaron un estudio empírico en Hong Kong, a 432 adolescentes entre varones y mujeres quienes fueron víctimas del acoso online, con el fin de determinar los niveles de victimización y el tipo de afrontamiento frente al acoso online. Entre los participantes, se concluye que, aquellos que viven en un ambiente prosocial-pasivo, presentan un comportamiento de afrontamiento activo, no obstante, los escolares que se encuentran en un ambiente prosocial-agresivo, cuentan con un comportamiento de afrontamiento evitativo, también se explica que la mayor prevalencia de represalias contra sus agresores, se da en el sexo masculino.

En Colombia, Baquero y Avendaño (2015)2\% mujeres y $45,8 \%$ hombres realizaron una investigación con el objetivo de diseñar un instrumento, en el cual analizaron las propiedades psicométricas para detectar el ciberbullying, se administró en 299 adolescentes el ( $45.8 \%$ hombres y $54.2 \%$ mujeres) en una edad de 15 años, el estudio es cuantitativo, con un diseño experimental de tipo instrumental y corte transversal, posee una adecuada consistencia interna con un (Alfa de Cronbach $\alpha=.864$ ), en el análisis factorial exploratorio se encontró tres factores que se identifican claramente en los ítems ligados a agresor, víctima y espectador, por otro lado, se manifiesta que el $21.74 \%$ de jóvenes se han visto comprometidos en el ciberbullying, sea como víctima y victimario.

En España, Cava y Buelga (2018) realizaron una investigación cuyo fin fue investigar las propiedades psicométricas de la Escala de Ciber-violencia en Parejas Adolescentes (Cib-VPA). Estas variables constan de dos dimensiones, la primera, ciberacoso perpetrado y la segunda, cibervictimización; cada una 
cuenta con 10 ítems que pretenden medir conductas agresivas y de control, que pueden ser realizadas o sufridas a través de redes sociales en parejas adolescentes. La muestra final, fue de 363 adolescentes (56.6\% mujeres y $43.3 \%$ varones). Mediante análisis factorial, se confirmaron dos factores similares en cada subescala: cibercontrol (conductas de excesivo control) y ciberagresión (amenazas e insultos a través de redes sociales). La fiabilidad de los dos factores se realizó mediante el cálculo del coeficiente omega, los resultaros fueron en la subescala de Ciberacoso perpetrado (cibercontrol .94 y ciberagresión .97) en la subescala Cibervictimización (cibercontrol .92 y ciberagresión .97). Existe correlación positiva entre las dimensiones cibercontrol y ciberagresión.

Para autores como Smith (2006, p.3), el ciberacoso es una extensión del maltrato, que constituye un hecho perpetrado y planificado, el cual se realiza de forma repetitiva y constante, mediante el uso de la web, teléfono móvil, por parte de un grupo de agresores o agresor contra una víctima que no puede defenderse. Se trata de un problema asociado a graves consecuencias, que pueden incurrir en daño físico, psicológico y social, entre niños y adolescentes Ministerio de Educación de Chile (MINEDUC, 2018).

Los principales actores del ciberacoso son, el agresor y la víctima; sin embargo, existen observadores, personas que son testigos (activos o pasivos) de la agresión. El cual es un entorno cercano, los que tienen el rol de asediar o intervenir en el este acto de hostigamiento. (Collell \& Escudé, 2004).

Para Salmerón, Blanco y Casas (2014, p. 269 ) el ciberacoso es el escenario donde la victima recibe agresiones de forma repetitiva y constante, por medio de aparatos digitales como; celulares, computadoras, etc. Con el fin de denigrar, lastimar y humillar al sujeto, logrando así, perturbar su autoestima, su equilibrio emocional y su entorno social. De la misma manera para Escobar-Echavarría (2017) el ciberacoso hace referencia a los comportamientos agresivos, amenazantes e intimidatorios que se da de forma persistente y sistemáticamente, asimismo el victimario no se muestra frontalmente y se oculta a través de medios electrónicos con el objetivo ridiculizar y afectar psicológicamente a una persona que es incapaz defenderse.
Para la Mineduc (2018) el ciberacoso también se da compartiendo información y contenido negativo, intimo, falaz, dañino, esto se realiza de forma anónima, sin el consentimiento de la víctima. Asimismo para Lwin (2012)especially for adolescents, who tend to engage in high-risk behavior online. Through the use of Protection Motivation Theory (PMT el ciberacoso se puede realizar de diferentes formas, como; compartir información ofensiva, ocasionar pleitos por medio de redes sociales, obtener información personal de la víctima para ser utilizada sin su consentimiento, la suplantación de otra persona en sus redes sociales, juegos en línea y correo electrónico, utilizando lenguaje inapropiado y ofensivo.

\section{A continuación, las siguientes formas en las que se realiza.}

Ciberbullying: Es el acoso, humillación, daño psicológico entre iguales por medio de internet (redes sociales, juegos en línea, etc.)

Grooming: El acoso por parte de un adulto por internet el cual se contacta con niños para ganarse su confianza con el fin de obtener satisfacción sexual.

Sexting: Esta se da en mandarse fotos y videos de carácter explícitamente sexual, el material es producido como parte de un juego entre la víctima y el acosador

Happy slapping: Maltratar a la víctima para poder exponerlo por medio de las redes sociales.

Ante esto hubo diferentes investigaciones para reducir la victimización del ciberacoso y así explican diferentes teorías, más la presente se basará en la teoría de la motivación protectora (PMT). (Roggers, 1975) esta indica la evaluación de las amenazas (gravedad percibida y la susceptibilidad percibida), la evaluación de afrontamiento (La eficacia de respuesta percibida y autoeficacia percibida) estos procesos cognitivos se activan cuando uno se enfrenta a una amenaza, que a su vez ayuda a reducir, para tener como fin una conducta (van Bavel, Rodríguez Priego, Vila, \& Briggs, 2019).

La evaluación de amenaza y evaluación de afrontamiento son procesos que actúan en conjunto para así tener una conducta final o de respuesta frente a un hecho que ponga en riesgo a la persona, hablamos sobre la motivación, 
para muchos autores los motivos que activan un comportamiento son variados y bastantes.

Según Bavel, Priego, Vila, \& Briggs (2019) la necesidad, carencia, deseo, temor y similares son estímulos que pueden romper o desequilibrar los esquemas de la estabilidad determinados en un individuo, ya sea en el nivel físico, social y psicológico, es así que en este momento la motivación actúa de manera ambigua, activa un comportamiento positivo o negativo.

Es así que Rippetoe y Rogers proponen en 1987, que la conducta surge de varias fuentes de información de carácter amenazante la cual estimula la subsiguiente estimación cognitiva, que a su vez dispone el cómo un individuo va a responder, partiendo de esta premisa es que la motivación protectora se fracciona intrínsecamente en dos dimensiones (Umeh, 2004, p4).

\section{La primera dimensión es la evaluación de las amenazas}

Según Salamanca y Giraldo (2011) la cual se entiende por la exposición de una serie de riesgos y vulnerabilidades ante distintas situaciones donde el miedo actúa como incentivo de la conducta, a la vez depende de su desarrollo alcanzado y la planeación del tal, busca tener la armonía entre el hombre y su bienestar, es así que identifica y evalúa las amenazas que causan un gran impacto para determinar una respuesta, dentro de estos encontramos dos sub dimensiones:

Gravedad percibida y la susceptibilidad percibida la cual resta a las ventajas de las respuestas des adaptativas la combinación de estas dos denomina la amenaza percibida; La gravedad percibida es el grado de seriedad que asume la persona frente a las diferentes amenazas y la susceptibilidad percibida, es la percepción individual del riesgo a contraer un peligro (Salamanca \& Giraldo, 201, p11).

Otros autores como Marin, Hoyos De los Rio, y Sierra (2019) mencionan que los factores de riesgo en las victimas de ciberbullyng tienen mucha relación con aspectos socio-familiares basados en experiencias de bullyng tradicional, deserción escolar, poco o ningún límite en cuanto a al uso de tecnologías digitales por sus padres, ningún apoyo social, soledad, discriminación racial, poca o ausente comunicación con sus padres, antecedentes de violación sexual en la infancia, tener pocas herramientas de sociabilización. En ese sentido la evaluación de las amenazas debe de ser congruente con las dificultades personales de la víctima en cuanto a sus necesidades de protección poco desarrollada.

Así mismo Del Río, Sádaba y Bringué (2010). Indica que se puede diferenciar dos tipos de riesgos; pasivos y activos, en los factores activos involucran anomalías en el uso de la tecnología sin que haya intención de los sujetos; por ejemplo, recibir mensajes acosadores o que personas no deseadas se contacten a través de internet o el celular. El simple hecho de tener una cuenta activa en una red social o jugar video juegos en línea a través de una computadora o Smartphone hace que los adolescentes se conviertan en víctimas potenciales de terceras personas que tienen intenciones nocivas. En cuanto al riesgo activo algunas personas que poseen determinadas actitudes frente al uso de la tecnología son presa fácil para los acosadores; por ejemplo, subir fotos privadas o comprometedoras a las redes sociales o brindar información excesiva y privada a personas desconocidas.

Por otra parte Sabater y López-Hernánez (2015) manifiestan en su investigación que el indicador más importante para la posibilidad del ciberacoso está relacionado con el tipo de privacidad de los acosados y acosadores, ellos tiene perfiles que son más públicos en el internet, comparten su información personal, fotos de ellos, amigos y familiares, asi mismo imitan y buscan fotografías eróticas en línea. Estos adolescentes tienden a interactuar de manera excesiva con extraños hasta inclusive pactan citas con ellos/as. Estos hábitos públicos son muy influyentes para ser acosador y acosado. Si se estudia en profundidad los factores de riesgo personales de las personas consideradas agresoras por ciberacoso cumplen las siguientes características; fueron víctimas de algún tipo acoso ya sea en internet o fuera de ella, sentirse amenazados, pensar que no tendrán consecuencias graves por sus actos en el internet, baja empatía, altos niveles de frustración, sufren episodios depresivos, poco autocontrol, baja autoestima, problemas de conducta.(González, 2016) 


\section{La segunda dimensión es la evaluación de afrontamiento}

Se define como el potencial o la probabilidad de éxito que percibe el individuo fundándose en situaciones en la que se podría dar frente a la amenaza, la cual estará conformada por una respuesta eficaz que influye en la reducción de la amenaza como una medida circunstancial y la autoeficacia que es la capacidad del individuo para tomar medidas orientadas a la prevención (Umeh, 2004, p4).

Según Piemontesi y Heredia (2009) Son las respuestas potenciales ante situaciones de conflicto y/o frustrantes, los cuales deben ser acorde a las posibilidades y recursos que cada persona posee, es decir, de qué manera se espera resolver la problemática, cuanto control y eficiencia se tiene ante los resultados cuáles serán las consecuencias y cuál será el camino correcto para resolverlo.

El afrontamiento también depende de las capacidades intrapersonales, como; la autoconfianza, automotivación y autoconcepto que caracteriza al sujeto, asimismo el ambiente interpersonal a niveles de autonomía, dependencia, creencias, valores, habilidades sociales y el apoyo de su entorno social, estas le proporcionan alternativas para contrarrestar situaciones amenazantes (Amarís, Camilo, Marcela, y Javier, 2013)

Son las respuestas potenciales ante situaciones de conflicto $y / o$ frustrantes, los cuales deben ser acorde a las posibilidades y recursos que cada persona posee, es decir, de qué manera se espera resolver la problemática, cuanto control y eficiencia se tiene ante los resultados cuáles serán las consecuencias y cuál será el camino correcto para resolverlo (Piemontesi \& Heredia, 2009).

Las estrategias para afrontar situaciones de conflicto depende de la visión y la evaluación que cada persona tiene y que significancia le da al conflicto, en la misma forma es dar valía a las opciones de afrontamiento y la estimación sobre los resultados, así esto se fortalece ante hechos que hayan ocurrido y considerar si estos episodios son tomados cómo desafíos o intimidaciones (Mora-Merchán, 2006)

Para Folkman (1986) existen dos tipos de afrontamiento el primero que es la valoración primaria, que es evaluar, considerar las consecuencias positivas y negativas, la valoración secundaria, son las capacidades que las personas tienen para darle frente, así mismo Salamanca y Giraldo (2011) manifiestan que estas respuestas cognitivas se agregan al sistema de creencias, originando resoluciones de carácter adaptativo o des-adaptativo, a su vez sometiéndose al grado de correspondencia o relación que el individuo encuentre entre la evaluación de amenaza y la evaluación de afrontamiento, y estarán guiadas por facilitadores o inhibidores de conducta, pertenecientes al entorno de la persona.

Por lo anteriormente expuesto, el objetivo general de esta investigación ha sido planteada y elaborada, con el fin de: establecer y validar las propiedades psicométricas de un instrumento, especialmente para evaluar las estrategias de afrontamiento frente a probables episodios de ciberacoso.

En los objetivos específicos: se pretende determinar los índices de validez de contenido, validez de constructo e índices de confiabilidad y por último la estandarización, que ayuden a catalogar este cuestionario como veraz y fiable para ser aplicado en distintas áreas de la Psicología.

\section{METODOLOGÍA}

El tipo de estudio es psicométrico, con un enfoque cuantitativo, de diseño no experimental de corte transversal, porque se realizara sin manipular deliberadamente la variable, (Hernández, 2014). La variable para la construcción de este instrumento se centra en analizar el comportamiento de afrontamiento de un adolescente frente al acoso online, por el cual se optará por construir y estandarizar para su uso en próximas investigaciones.

\section{Participantes}

Para definir el tipo de muestra de la investigación se utilizó la técnica de muestreo no probabilístico (por conveniencia) la cual según Otzen y Manterola (2017) Admite escoger a aquellos individuos que accedan a ser incluidos en la investigación. Esto fundamentado en la conveniencia de accesibilidad y proximidad de los individuos para el investigador. 
La prueba piloto se aplicó a 250 adolescentes entre 14 y 17 años de edad, que cursaban el 4to y 5 to año de secundaria de una Institución Educativa de la ciudad de Juliaca, con la intensión de saber si los ítems tenían una adecuada comprensión, si se ajustaba al contexto y si eran acorde a la edad de los participantes.

La aplicación masiva estuvo conformada por 300 adolescentes del departamento de Puno, de tres Instituciones Educativas públicas y privadas del nivel secundario, así mismo sus edades oscilan entre 12 y 17 años, de ambos sexos que tienen servicio de internet en casa o datos móviles, con una subscripción activa a redes sociales o juegos en línea. Esta aplicación se realizó de manera virtual por medio de plataforma Google formularios, donde se encontraba el consentimiento informado antes de tener acceso al cuestionario.

\section{Instrumentos}

Para Soriano (2014) los instrumentos son herramientas útiles, de modo que nos permitan obtener datos, se asignan de manera numérica y se cuantifican, cabe señalar que deben tener un proceso de confiabilidad y validez del constructo.

El siguiente instrumento inicialmente estuvo compuesta por 18 ítems, conformado por dos dimensiones con el objetivo de categorizar las estrategias de afrontamientos frente al ciberacoso en adolescentes de 12 a 17 años, el cual fue sometido a un análisis de validez del constructo realizado por 6 jueces expertos en la materia, en base a sus opiniones sé hizo las correcciones y cambios en los sentidos de las preguntas, como resultado el cuestionario está compuesto por 15 ítems, con formato de escala Likert de 5 alternativas "nunca", "casi nunca", "de vez en cuando", "casi siempre" y "siempre".

\section{Dimensión 1:}

\section{Evaluación de las amenazas}

Está compuesta por 9 ítems, las cuales son: 1 , $4,5,6,9,10,11,12$ y 13 estos ítems refieren a la predisposición que los adolescentes pueden tener ante situaciones amenazantes de ciberacoso.

\section{Dimensión 2:}

\section{Evaluación de afrontamiento}

Está compuesta por 6 ítems, los cuales son; $2,3,7,8,14$ y 15 estos ítems refieren a las estrategias y comportamientos que asumen los adolescentes para resolver y/o evitar ser víctima de ciberacoso, además cuáles son sus respuestas de prevención ante dichas situaciones.

\section{Evaluación de afrontamiento}

Está compuesta por 6 ítems, los cuales son; $2,3,7,8,14$ y 15 estos ítems refieren a las estrategias y comportamientos que asumen los adolescentes para resolver y/o evitar ser víctima de ciberacoso, además cuáles son sus respuestas de prevención ante dichas situaciones.

\section{Análisis de datos}

Para iniciar el análisis de datos se sometió la escala al juicio experto de 6 jueces que fueron elegidos por su especialidad, experiencia en el tema y grado académico, los cuales dieron algunas sugerencias que los investigadores tomamos en cuenta para la elaboración final de los ítems. Seguidamente se recolecto las respuestas de los participantes de la plataforma de encuesta de Google y se prosiguió a vaciar los datos en el programa estadístico Excel. Para el análisis factorial e interpretación de los resultados que a continuación se muestra en las tablas pertinentes se utilizó el software estadístico SPSS, para realizar la estructura factorial, se realizó mediante IBM AMOS.

\section{RESULTADOS}

\section{Análisis De Validez}

En la tabla 1 se puede observar los resultados del análisis de validez de contenido mediante el coeficiente V. Aiken, el cual se realizó a cargo de 6 jueces especialistas en el tema. Los cuales hicieron sugerencias y comentarios acerca de cada ítem, los cuales fueron aceptados se evaluaron criterios como; claridad, congruencia y contexto obtuvieron un coeficiente de .98 el cual indica que el instrumento posee una excelente validez. 
Tabla 1

Tabla de validez de jueces por $V$ de Aiken

$\begin{array}{llll}\text { Criterios } & \text { Expertos } & \text { Sub total } & \text { Interpretación } \\ \text { Claridad } & 6 & 0.97 & \text { Excelente validez } \\ \text { Congruencia } & 6 & 0.98 & \text { Excelente validez } \\ \text { Contexto } & 6 & 0.98 & \text { Excelente validez } \\ \text { Dominio de constructo } & 6 & 0.98 & \text { Excelente validez } \\ \text { Análisis de validez según } & & 0.98 & \text { Excelente validez } \\ \text { el coeficiente de V. de } & & & \\ \text { Aiken } & & \end{array}$

Validez de constructor o análisis factorial confirmatorio

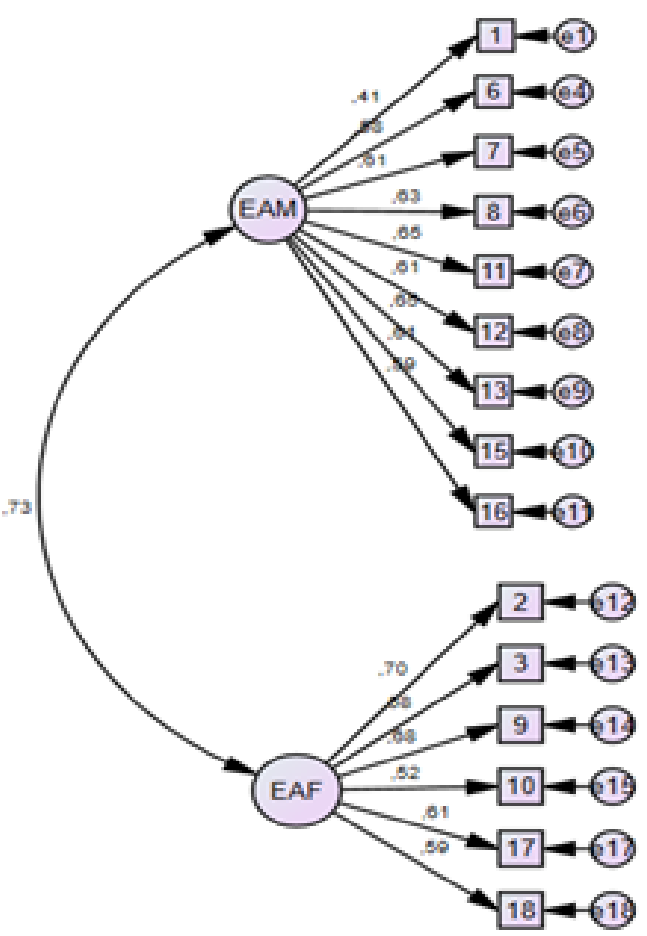

Figura 1

Modelo de ecuaciones estructurales basado en dos factores

Para el análisis del constructo, el instrumento se aplicó una población de $n=300$ sujetos. La medida de adecuación de muestreo $(\mathrm{KMO}=0,876)$ indica que adecuada intercorrelación de ítems, aunado a ello, la significancia crítica de Barlett es menor de 0,05 , todo ello indica que el análisis factorial es coherente con la naturaleza del instrumento. Presentando el modelo dos factores se logra explicar el 45,6 \% de la varianza que genera el instrumento. La matriz de componente rotado corrobora la estructura factorial, siendo que la dimensión evaluación de la amenaza se configura por lo ítems $1,4,5,6,9.10 .11 .12$ y 13 la dimensión evaluación de afrontamiento tiene a los ítems 2, 3, 7, 14 y 15 Esta estructura presenta un adecuado ajuste de modelo siendo el $C F I=0,882, T L I=0,860$ y RMSEA $=0,077$.

\section{Confiabilidad}

En la tabla 3 se puede observar el coeficiente de Alfa de Cronbach el cual permite valorar la fiabilidad del cuestionario por medio del conjunto de ítems, como resultados obtuvimos .873 el 
cual presenta un nivel de confiabilidad bueno, de acuerdo a los criterios para evaluar (George \& Mallery, 2003)

Así mismo se analizó la fiabilidad de las dos dimensiones que presenta este instrumento mediante el cálculo del coeficiente omega de McDonald's. La dimensión: Evaluación de las amenazas obtuvo una fiabilidad de .957 y la dimensión: Evaluación de afrontamiento obtuvo una fiabilidad de .851 como se muestra en la tabla 4.

Tabla 3

Análisis de fiabilidad por Alfa de Cronbach

Alfa de Cronbach N de elementos

$.873 \quad 15$

Tabla 4

Análisis de fiabilidad por Omega de McDonald's

\begin{tabular}{ll} 
Dimensión & McDonald's w \\
Evaluación de amenaza & .957 \\
Evaluación de afrontamiento & .851 \\
\hline
\end{tabular}

En la tabla 5 se puede el análisis de confiabilidad, para este se utilizó el Alfa de Cronbach en los cuales se considera según George y Mallery (2003) los valores > o = a .7 obtienen una fiabilidad aceptable, por ende, cada ítem es aceptable.

Tabla 5

Análisis de fiabilidad por ítems

\begin{tabular}{lllll} 
& $\begin{array}{l}\text { Media de escala si el } \\
\text { elemento se ha supri- } \\
\text { mido }\end{array}$ & $\begin{array}{l}\text { Varianza de escala } \\
\text { si el elemento se ha } \\
\text { suprimido }\end{array}$ & $\begin{array}{l}\text { Correlación total } \\
\text { de elementos co- } \\
\text { rregida }\end{array}$ & $\begin{array}{l}\text { Alfa de Cronbach si } \\
\text { el elemento se ha } \\
\text { suprimido }\end{array}$ \\
\hline Item1 & 42,88 & 51,945 &, 390 &, 871 \\
Item2 & 42,55 & 49,834 &, 553 &, 863 \\
Item3 & 42,57 & 50,527 &, 489 &, 866 \\
Item4 & 42,49 & 50,425 &, 509 &, 865 \\
Item5 & 42,50 & 50,177 &, 535 &, 864 \\
Item6 & 42,53 & 49,420 &, 560 &, 863 \\
Item7 & 42,55 & 50,041 &, 544 &, 864 \\
Item8 & 42,47 & 51,681 &, 466 &, 867 \\
Item9 & 42,55 & 49,880 &, 567 &, 863 \\
Item10 & 42,46 & 50,102 &, 526 &, 865 \\
Item11 & 42,56 & 49,458 &, 586 &, 863 \\
Item12 & 42,68 & 49,549 &, 566 &, 865 \\
Item13 & 42,46 & 50,062 &, 514 &, 862 \\
Item14 & 42,57 & 49,657 &, 571 &, 869 \\
Item15 & 42,77 & 50,586 &, 442 & \\
\hline
\end{tabular}




\section{Baremación}

En la tabla 6 se observa la tabla de percentil en el cual se elaboró la baremación de este cuestionario a partir de su aplicación en 300 sujetos, distribuidos en 3 cortes donde según los percentiles convertidos a puntuaciones dan los siguientes resultados de 0-39, el encuestado tiene un bajo nivel de afrontamiento; si el puntaje es de 40-49, el encuestado tiene un mediano nivel de afrontamiento y una puntuación de 50 a 75 significa que el encuestado presenta un alto nivel de afrontamiento. Cuanto más alto es el puntaje, mayor será el nivel de afrontamiento al ciber acoso.

Tabla 6

Tabla Percentil con valores categóricos en 3 cortes

\begin{tabular}{lllcl} 
Pc & Evaluación de amenazas & Evaluación de Afrontamiento & Total & Nivel \\
\hline $5-25$ & $0-23$ & $0-15$ & $0-39$ & Bajo \\
$30-70$ & $24-29$ & $16-20$ & $40-49$ & Medio \\
$75-99$ & $30-42$ & $21-28$ & $50-75$ & Alto \\
\hline
\end{tabular}

\section{DISCUSIÓN}

El ciberacoso hoy en día es un término que está siendo muy utilizado ya que en la actualidad son más los niños y adolescentes que hacen uso de los Smartphone y computadoras con acceso al internet, exponiéndose a muchos peligros entre ellos al acoso en línea ya sea de personas de su entorno o de desconocidos que usan estas herramientas tecnológicas con fines oscuros o delictivos.

La validación del cuestionario fue realizada por profesionales especialistas voluntarios y obtuvo aceptables valores de consistencia interna, un $\mathrm{V}$ de Aiken $=.98$. Además, se realizó el análisis factorial exploratorio por KMO obteniendo el resultado de .876 lo que significaría que el nivel de significancia es óptimo dentro de un análisis factorial exploratorio y confirmatorio para proseguir con el análisis de confiabilidad de la escala, después del análisis respectivo se obtuvo; como coeficiente .873 lo que significa que el instrumento tiene una confiabilidad óptima. La cual es comparada con otros estudios hallados, Higuita-gutiérrez \& Cardonaarias (2017) realizaron una investigación con la finalidad de validar una escala de bullying en adolescentes de instituciones educativas de Medellín, Colombia, mediante un estudio de diseño instrumental y de tipo psicométrico, no experimental con la participación de 3460 adolescentes, entre varones y mujeres de 16 años de edad, obteniendo como resultado que el instrumento tiene un grado adecuado de confiabilidad de, 80 , el cual se obtuvo a través del Alfa de Cronbach. El cual es inferior al de la investigación teniendo en cuenta que la población es mucho mayor en dicha investigación.

Por su parte Baquero y Avendaño (2015)2\% mujeres y $45,8 \%$ hombres efectuaron una investigación que tenía como objetivo diseñar un instrumento y analizar las propiedades psicométricas para detectar el ciberbullying, esta se aplicó en 299 adolescentes (54.2\% mujeres y $45.8 \%$ hombres) de 15 años de edad, el estudio es cuantitativo, con un diseño experimental de tipo instrumental y corte transversal, esta obtuvo una adecuada consistencia interna con un (Alfa de Cronbach $\alpha=.864$ ) el cual es similar a la investigación en confiabilidad y población de estudio.

Así mismo López (2016) realizo una investigación en México similar titulada "Propiedades Psicométricas De Un Instrumento De Acoso Cibernético En Estudiantes Universitarios Mexicanos 2018" el cual obtuvo la distribución factorial una escala de victimización través del celular y la web se aplica a 170 estudiantes de una institución pública de Morelos de las facultades de psicología, medicina, derecho y administración. El alfa de Cronbach obtenidos en este estudio para ambos factores fue más baja (.65 para el factor Móvil y .63 para el factor Internet). Por lo tanto, estos valores son menores a los obtenidos en la presente investigación. 
Por otra parte, Arce, Velasco, Novo, y Fariña (2014) diseñó un estudio con el objetivo de desarrollar una escala de evaluación del acoso escolar en adolescentes. Mediante un estudio de tipo no experimental con corte descriptivo con una muestra de 308 adolescentes, el $52.7 \%$ chicas, con edades entre los 12 y los 17 años que cursaban la ESO. Los resultados concretaron una escala compuesta por 26 ítems y 4 factores (acoso psicológico, exclusión, acoso físico y acoso relacional) que se mostró fiable tanto en el total de la escala, $\alpha=.95$, El cual es un valor superior al del presente estudio teniendo en cuenta que la población de estudio es inferior en ambas investigaciones.

Es por ello que la utilización del "cuestionario de estrategias de afrontamiento al ciberacoso en adolescentes" para medir niveles de afrontamiento frente al ciberacoso, es totalmente viable y recomendable para su uso profesional ya que a comparación de otros estudios similares tiene un adecuado nivel de confiabilidad y al ser un instrumento de magnitud completa para el enfoque actual, ya el ciberacoso no solo estará presente durante el periodo de clases no presenciales sino que se mantendrá en el tiempo debido a que la utilización de estas herramientas tecnológicas serán utilizados constantemente por los adolescentes e incluso individuos de otras edades.

La limitación del presente estudio estaría en la forma y el periodo de la aplicación del instrumento ya que este fue aplicado de manera virtual mediante plataformas digitales y en periodo de clases no presenciales, se tendría que analizar el comportamiento de este cuestionario cuando las clases sean presenciales y la aplicación sea de manera también presencial.

Se logra construir el Cuestionario de Estrategias de Afrontamiento al Ciberacoso, la cual fue sometida a estudios para analizar su fiabilidad y confiabilidad. En el misma que se muestra la bondad del instrumento, el cual se detalla a continuación:

Se halló un Alfa de Cronbach de .873 y las 2 dimensiones obtuvieron un coeficiente de omega de .957 y. 851 respectivamente, lo que demuestra que el instrumento tiene una confiabilidad adecuada, además fue sometido a una revisión de 4 criterios, por 6 jueces especialistas en el tema, mediante $\mathrm{V}$ de Aiken, el cual se obtiene un puntaje 0.98 por tal el instrumento tiene una apropiada validez de contenido.

El análisis factorial confirmatorio del instrumento, se una varianza total de $45.6 \%$ el cual nos indica que el cuestionario funciona con 2 factores, propuestos teóricamente (evaluación de amenazas y evaluación de afrontamiento), asimismo en el análisis factorial confirmatorio prueba de Kaiser Meyer Elkin ,876 y la prueba de esfericidad con una sig. de .000 , por el cual los ítems se relacionan y pueden formar factores, asimismo se realiza la estructura factorial de la matriz la cual presenta un adecuado ajuste de modelo siendo el $C F I=0,882, T L I=0,860$ y RMSEA $=0,077$, por el cual se considera que dicha investigación se puede trabajar con más de dos factores.

Se logra establecer y validar las propiedades psicométricas de un instrumento, especialmente para evaluar las estrategias de afrontamiento frente a probables episodios de ciberacoso. Este cuestionario es válido y confiable para su aplicación en distintas áreas de la Psicología.

\section{Declaración de financiamiento y de conflictos de interés:}

El estudio fue financiado por los autores, quienes declaran no tener conflictos de interés

Correspondencia:

Eddy Wildmar Aquize Ancob

Correo electrónico:

eddy.wildmar@upeu.edu.pe 


\section{REFERENCIAS}

Amarís, M. M., Camilo, M. O., Marcela, V. A., \& Javier, Z. (2013). Estrategias de afrontamiento individual y familiar frente a situaciones de estres psicologico. Psicología Desde El Caribe, 30(1), 123-145. https://www.redalyc.org/ pdf/213/21328600007.pdf

Albornoz, D. (27 de agosto de 2020). Hiperderecho. https://hiperderecho.org/2020/08/que-nos-dicenlos-datos-de-no-al-acoso-virtual-sobre-lascaracteristicas-de-la-violencia-de-genero-enlinea-en-el-peru/

Álvarez-G, D., Nuñez, J.C., Álvarez, L., Dobarro, A., Rodríguez, C., \& GonzálezCastro, P. (2011). Violencia a través de las tecnologías de la información y la comunicación en estudiantes de secundaria. Anales de Psicología, 27, 221-231. Revista anales de psicologia. https://revistas. um.es/analesps/article/view/113661.

Arce, R., Velasco, J., Novo, M., \& Fariña, F. (2014). Elaboración y validación de una escala para la evaluación del acoso escolar. Revista Iberoamericana de Psicología Y Salud, 5(1), 71-104. http://www.redalyc.org/articulo. oa? id=245129173005

Caba, M-J \& B, S (2018) Propiedades psicométricas de la Escala de Ciber-Violencia. Revista suma psicologia. http://www.scielo.org.co/pdf/sumps/ v25n1/0121-4381-sumps-25-01-51.pdf

Baquero, A., \& Avendaño, B. L. (2015). Diseño y análisis psicométrico de un instrumento para detectar presencia de ciberbullying en un contexto escolar. Psychology, Society, \& Education, 7(2), 213. https://doi.org/10.25115/psye.v7i2.534

Chan, H. C. (Oliver), y Wong, D. S. W. (2017). Coping with cyberbullying victimization: An exploratory study of Chinese adolescents in Hong Kong. International Journal of Law, Crime and Justice, 50, 71-82. https://doi.org/10.1016/j. ijlcj.2017.04.003

Del Rio, J., Sádaba, C. \& Bringué, X. (2010). Menores y redes ¿sociales?: de la Amistad al ciberbullying. Revista de Estudios de Juventud, 88, 115- 130. Injuve, Madrid. http://www.injuve.es/sites/default/ files/RJ88-09.pdf

Escobar E, J., Montoya González, L. E., Restrepo Bernal, D., \& Mejía Rodríguez, D. (2017). Cyberbullying and Suicidal Behavior: What is the Connection? About a Case. Revista Colombiana de Psiquiatria, 46(4), 247-251. https://doi. org/10.1016/j.rcp.2016.08.004

Folkman, S., Lazarus, R. S., Gruen, R. J., \& DeLongis, A. (1986). Appraisal, Coping, Health Status, and Psychological Symptoms. Journal of Personality and Social Psychology, 50(3), 571-579. https:// doi.org/10.1037/0022-3514.50.3.571

González G, A. (2016). Factores de riesgo en el ciberacoso: revisión sistemática a partir del modelo del triple riesgo delictivo ( TRD ). IDP. Revista de Internet, Derecho Y Política, 22, 62-81. http://www.redalyc.org/articulo. oa?id=78846481006

Hernández, R. (2014). Metodología de la investigación. (I. E. DE D.V., Ed.) (sexta edic). Mexico.

Higuita, L. F., \& Cardona-arias, J. A. (2017). Validación de una escala de bullying en adolescentes de instituciones educativas de Medellín, Colombia Educación Y Educadores, 20(1), 9-23. https:// doi.org/10.5294/edu.2017.20.1.1

López, M. G. (2016). Propiedades Psicométricas De Un Instrumento De Acoso Cibernético En Estudiantes Universitarios Mexicanos. Revista Electrónica "Actualidades Investigativas En Educación, 15(1), 1-19. https://www.scielo.sa.cr/ pdf/aie/v16n1/1409-4703-aie-16-01-00091.pdf.

Lwin, M. O., Li, B., \& Ang, R. P. (2012). Stop bugging me: An examination of adolescents' protection behavior against online harassment. Journal of Adolescence. https://doi.org/10.1016/j. adolescence.2011.06.007

Marin , A. F., Hoyos De los Rio, O. L., \& Sierra Pérez, A. (2019). Factores De Riesgo Y Factores Con El Ciberbullying Entre Adolescentes: Una Revisión Sistemática. Papeles Del Psicólogo, 40(2), 108127. https://doi.org/https://doi.org/10.23923/pap. psicol2019.2899 PDF

Mineduc, (2018). CIBERACOSO: Centro de Estudios Mineduc.

Mora, J. (2006). Las estrategias de afrontamiento, ¿mediadoras de los efectos a largo plazo de las víctimas del bullying? Anuario de Psicología Clínica $Y$ de La Salud = Annuary of Clinical and Health Psychology, 2(2), 15-26. http:// institucional.us.es/apcs/doc/APCS_2_esp.pdf.

Otzen, T., \& Manterola, C. (2017). Técnicas de Muestreo sobre una Población a Estudio. Int. J. Morphol, 35(1), 227-232. https:// scielo. conicyt. cl/scielo. php? pid=S071795022017000100037\&script=sci_abstract.

Perú21. (2016). Cuidado con el "grooming", el acoso sexual a menores en internet [Video] | Lima | Peru21.

Perú21. (2017). Ciberbullying crece en el Perú pero la mayoría no lo denuncia | Lima | Peru21. 
Piemontesi, S. E., \& Heredia, D. E. (2009). Afrontamiento ante exámenes: Desarrollos de los principales modelos teóricos para su definición y medición. Revista Anales de Psicologia, 25(1), 102-111. https://revistas.um.es/analesps/article/ view/71551.

Sabater F, C., \& López-Hernánez, L. (2015). Factores de Riesgo en el Ciberbullying. Frecuencia y Exposición de los Datos Personales en Internet. Revista Internacional de Sociología de La Educación, 4(1), 1-25. https://doi.org/10.4471/ rise.2015.01

Salmerón R, M. A., Blanco Sánchez, A. I., \& Casas Rivero, J. (2014). Hablemos de Ciberacoso y adolescentes. An Pediatr Contin, 12(5), 269-273. https://cipesalud.com.ar/wp-content/ uploads/2021/04/ciberacoso.pdf.
Soriano, A. M. (2014). Diseño y validación de instrumentos de medición. Design and validation of measurement instruments. Diá-Logos 14, 8(13), 19-40. https://www.lamjol.info/index.php/ DIALOGOS/article/view/2202.

Villegas R, I. M. (2019). Adaptación de la Escala de Conductas del Sexting en Adolescentes de Instituciones Educativas Públicas del distrito de Lurigancho Chosica 2019 0-3. Obtenido de https://repositorio.ucv.edu.pe/bitstream/ handle/20.500.12692/39398/Villegas_RIM. pdf?sequence=1\&isAllowed

Recibido: $11 / 07 / 2021$

Aceptado: 20/10/2021 